\title{
A case of bilateral thalamic gliomas coexisting with status epilepticus: a rare association
}

Yeşim Güzey Aras ${ }^{1}$, Dilcan Kotann ${ }^{1}$, Davut Ceylan², Nimet Uçaroğlu Can ${ }^{1}$, Ayhan Bölük ${ }^{3}$, Süheyla Uyar Bozkurt ${ }^{4}$

${ }^{1}$ Department of Neurology, Sakarya University Training and Research Hospital, Sakarya, Turkey

2Department of Neurosurgery, Sakarya University Training and Research Hospital, Sakarya, Turkey

${ }^{3}$ Department of Neurology, Sakarya University Hospital, Sakarya, Turkey

${ }^{4}$ Department of Pathology, Marmara University Training and Research Hospital, Istanbul, Turkey

Submitted: 30 May 2016

Accepted: 13 June 2016

Arch Med Sci Civil Dis 2016; 1: e51-e54

DOI: 10.5114 /amscd.2016.60820

Copyright $\odot 2016$ Termedia \& Banach

We have observed a male patient with bilateral thalamic gliomas with epileptic seizures. Here, we would to like comment on this rare observed case.

Bilateral thalamic gliomas are rare tumors of the central nervous system, which are usually unilateral [1]. Primary thalamic gliomas include a different subgroup called bilateral thalamic gliomas, and these tumors involve the thalami bilaterally. They form a sizable mass lesion in the thalami. Their clinical course may include a variety of conditions including behavioral problems, personality changes, dementia and motor and sensory loss [2]. Cranial magnetic resonance imaging (MRI) reveals a hyperintense image in T2 and FLAIR sections and an isointense image in T1 section without contrast uptake [3]. Definitive diagnosis of a tumor is made by histopathological examination of tissue obtained by stereotactic biopsy. Despite treatment, its prognosis is rather bad [4]. In this paper, we describe a male patient who was diagnosed with primary bilateral thalamic astrocytic glial tumors using imaging and biopsy upon presentation with complaints of progressive weakness in the left arm and leg and who died after status epilepticus.

A 55-year-old male patient was admitted to the emergency department with complaints of progressive weakness in his left arm and leg. From his history, it was learned that his complaints began 4 months ago, that he had been constantly steady recently and that his gait was impaired in the recent period. His medical history was unremarkable, except hypertension, diabetes and coronary artery disease. He had no significant family history. His neurological examination revealed apathetic appearance, difficulty in cooperation, mild hemiparesis in the left arm and leg, hemihypoesthesia on the left, normal left deep tendon reflexes and an unremarkable response in bilateral soles. In his brain computed tomography (CT), both thalami were isodense; however, they were found to be enlarged into the ventricles (Figure 1). Cranial MRI revealed an image consistent with a mass lesion with edematous periphery extending into the ventricle, which was isointense in T1 sections and hyperintense in T2 sections, and linear contrast enhancement (Figures $2 \mathrm{~A}, \mathrm{~B}$ ). Diffusion MRI also revealed limited diffusion in both thalami, which was significant

\author{
Corresponding author: \\ Dilcan Kotan \\ Department of Neurology \\ Sakarya University Training \\ and Research Hospital \\ 25050 Erzurum, Turkey \\ Phone: 4882143030 \\ E-mail: dilcankotan@yahoo. \\ com
}




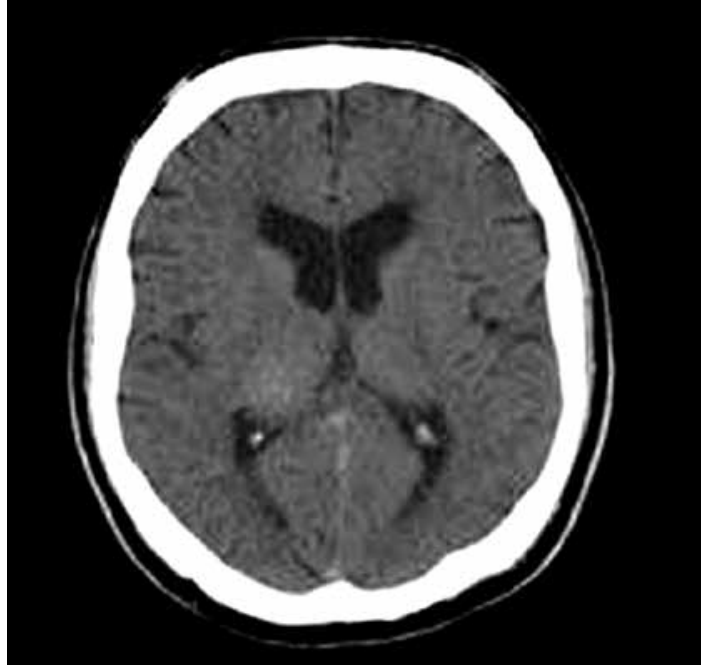

Figure 1. In brain CT, both thalami are isodense and their enlargement into ventricles is apparent
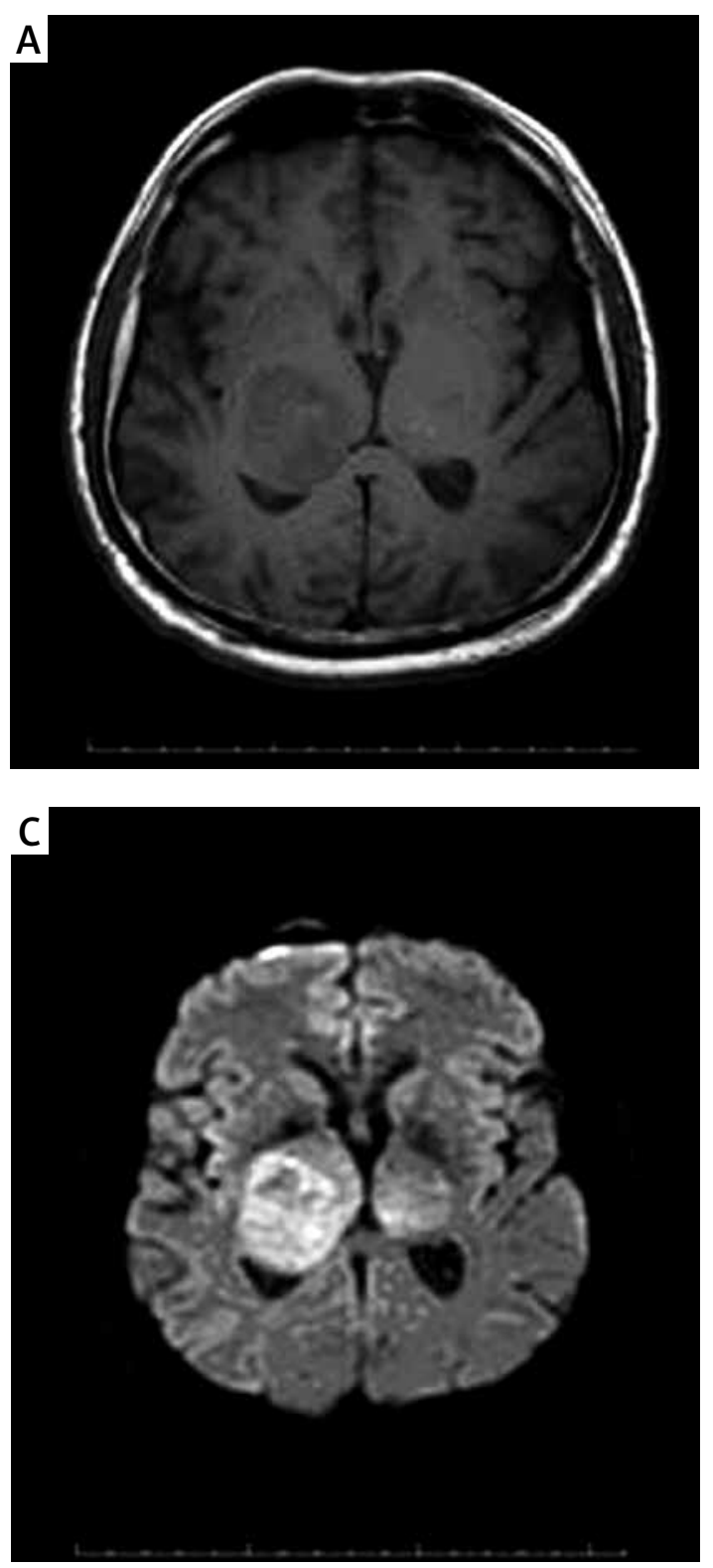

on the right (Figure $2 \mathrm{C}$ ). His histopathological examination performed after stereotactic biopsy of suspected tumor revealed an astrocytic neoplasm with oval nucleus and eosinophilic cytoplasm showing atypia and pleomorphism in hematoxylin and eosin staining, and the neoplasm was assessed as a glial tumor with high-grade vascular proliferation and necrotic areas (Figures 3 A-D). While he was being referred for treatment, he was taken to the intensive care unit with an epileptic seizure followed by mental confusion. During his examination, he was confused, disoriented, localized his left side less to painful stimuli and his bilateral sole reflexes were extensor. It was not possible to control his seizures despite antiepileptic and antiedema treatment, and on the third day of follow-up he was taken for mechanical ventilation due to status epilepticus and died on the tenth day of follow-up.

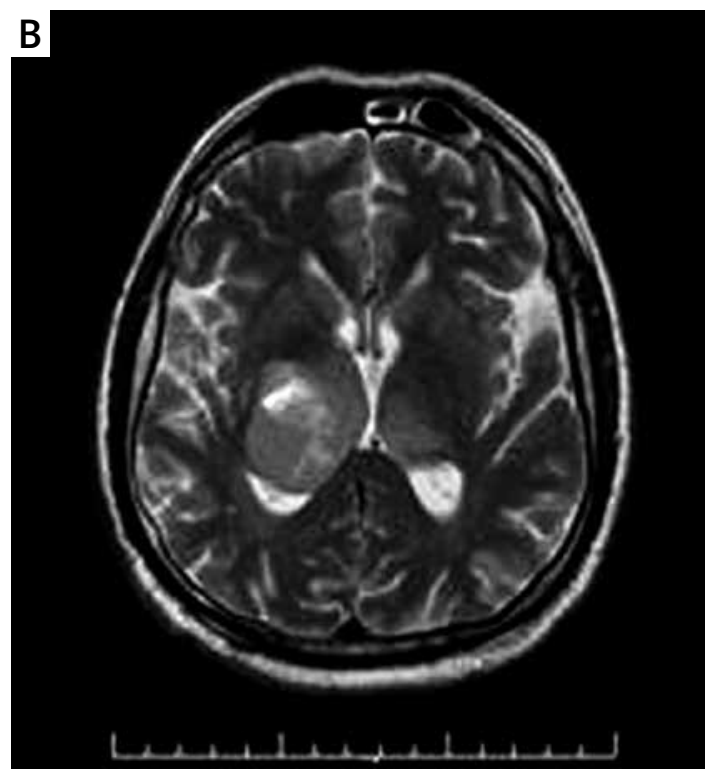

Figure 2. Isodense image in cranial MRI T1-weighted sections (A), In T2-weighted axial section, hyperintense image consistent with a mass lesion with edematous periphery extending into ventricle (B), limited diffusion in both thalami in diffusion MRI (C) 

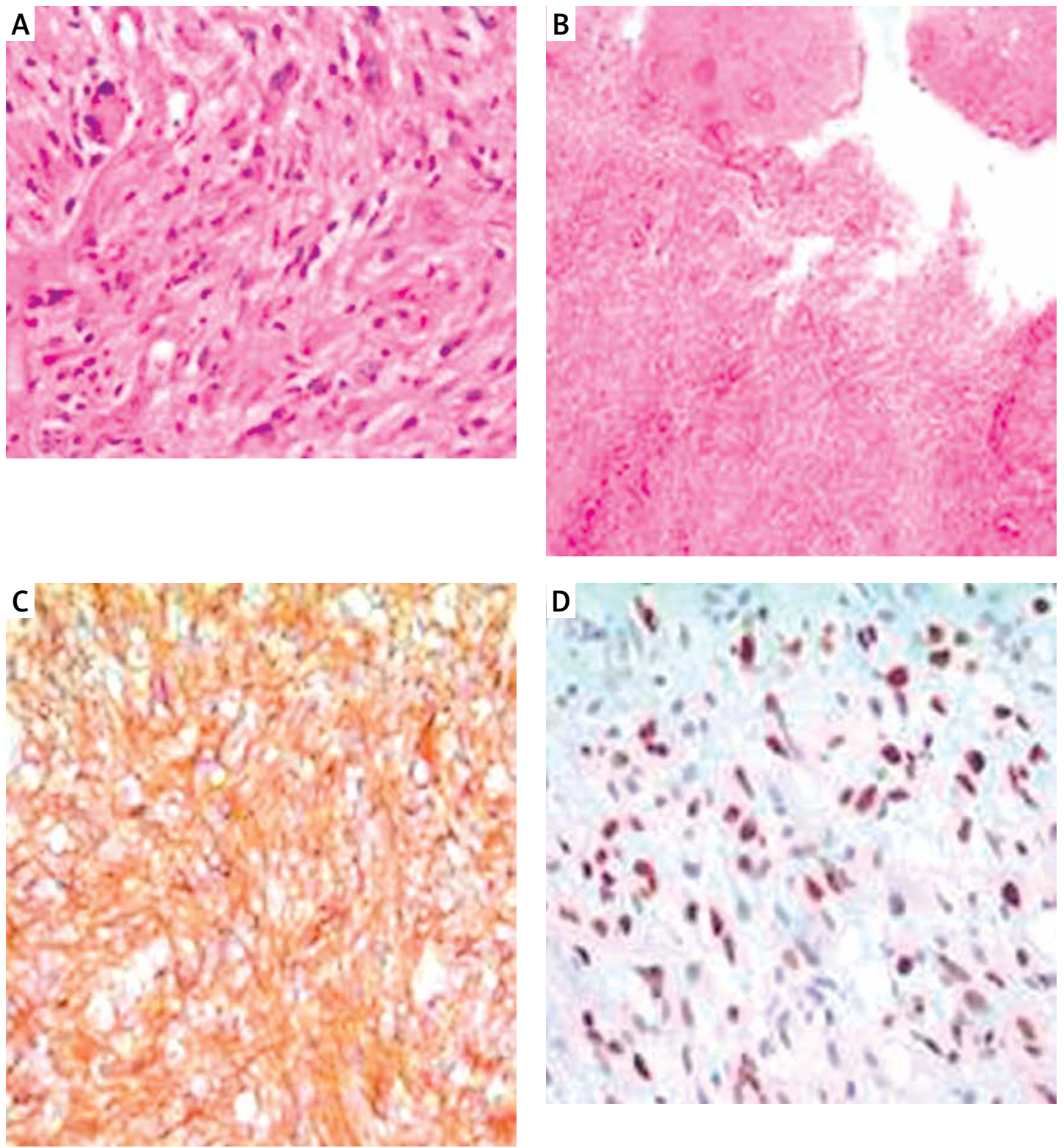

Figure 3 A-D. Astrocytic neoplasm with oval nucleus and eosinophilic cytoplasm showing atypia and pleomorphism, and vascular proliferation and necrotic area in neoplasm $(H+E, 400 x)$. Diffuse and strong immune reactivity in neoplastic cells (GFAP 400x). Increased proliferation in neoplastic cells (Ki67, 400×)

Bilateral thalamic gliomas are rare tumors, which are common between the ages of 8 and 70 , and the average age is around 39 years; its incidence is equal in females and males [4]. Our case was a 55 -year-old man. In view of the literature, there are a limited number of case reports and small case series. The hypothesis that best describes bilateralness of these tumors states that the tumor starts unilaterally and passes to the opposite thalamus through the prerubral part of the midbrain, posterior section of the corpus callosum and massa intermedia. Another hypothesis states that the tumor originates from the subependymal section of the third ventricle and affects bilateral thalami bilateral spread [3]. These patients often clinically present with symptoms including memory and personality changes and increased intracranial pressure (IICP) [2]. Hemiparesis was reported in approximately half of the cases with one-sided thalamic tumor [5]. Although our patient had bilateral thalamic tumors, he presented with hemiparesis and developed cognitive impairment thereafter.

Prognosis of these tumors is rather bad; the mean time between emergence of symptoms and death is 1 year [4]. Our patient died 6 months after developing the symptoms and slowly progressing hemiparesis. In his follow-up, he was found to also have developed cognitive impairment, and he differed from the cases in the literature with his antiepileptic treatment-resistant seizures and status epilepticus. Douis et al. reported a patient who 
presented with epileptic seizure, as in our case [6]. IICP symptoms and headache are less common in bilateral thalamic tumors, whereas they are more common in unilateral thalamic tumors [4]. Our patient had no IICP findings or headache. It is extremely important to radiologically distinguish bilateral thalamic lesions from other pathologies. Brain CT reveals a bilateral symmetric isodense lesion without contrast uptake. Brain CT of our patient revealed swelling and an isodense image in the bilateral thalami. In cranial magnetic resonance imaging, bilateral thalamic tumors are homogeneous isointense in T1-weighted images and hyperintense in T2-weighted and FLAIR images [7]. Similar radiological characteristics were observed for our case. The definitive diagnosis is made by histopathological examination. The histopathological examination of our patient carried out after stereotactic biopsy revealed high-grade astrocytic glial tumors. However, while our patient was being referred for treatment, he died. Here, we present a rare case diagnosed as highgrade astrocytic glial tumor by histopathological examination after imaging revealed an extensive bilateral thalamic lesion. Clinicians should bear in mind that such patients may develop treatment-resistant epileptic seizures, which could be a factor responsible for mortality.

\section{Conflict of interest}

The authors declare no conflict of interest.

\section{References}

1. Cheek WR, Taveras M. Thalamic tumors. J Neurosurg 1996; 24: 505-13.

2. Uchino M, Kitajima S, Miyazaki C, Shibata I, Miura M. Bilateral thalamic glioma case report. Neurol Med Chir 2002; 42: 443-6.

3. Menon G, Naır S, Sudhir J, Rao BRM, Krıshnakumar K. Bilateral thalamic lesions. Br J Neurosurg 2010; 24: 566-71.

4. Benbir G, Sayılır I, Öz B, Uzan M, Yalçınkaya C. Bilateral thalamic glioma: a case report. J Neurol Sci 2008; 25: 301-5.

5. Beks JWF, Bouma GJ, Journee HL. Tumors of the thalamic region. A retrospective study of 27 cases. Acta Neurochir (Wien) 1987; 85: 125-7.

6. Douis H, Jafri $M$, Sherlala K. Bilateral thalamic glioma. Arch Neurol 2008; 65: 1666-7.

7. Partlow GD, del Carpio-O'Donovan R, Melanson D, Peters TM. Bilateral thalamic glioma: review of eight cases with personality change and mental deterioration. Am J Neuroradiol 1992; 13: 1225-30. 